11,13

\title{
Динамика развития канала переключения в планарных структурах на основе диоксида ванадия
}

\author{
( М.А. Беляев ${ }^{1}$, П.П. Борисков ${ }^{1}$, А.А. Величко ${ }^{1}$, А.Л. Пергамент ${ }^{1}$, В.В. Путролайнен ${ }^{1}$, \\ Д.В. Рябоконь ${ }^{2,3}$, Г.Б. Стефранович ${ }^{1}$, В.И. Сысун ${ }^{1}$, С.Д. Ханин ${ }^{2,3}$ \\ ${ }^{1}$ Петрозаводский государственный университет, \\ Петрозаводск, Россия \\ ${ }^{2}$ Военная академия связи им. маршала СССР С.М. Буденного, \\ Санкт-Петербург, Россия \\ ${ }^{3}$ Российский государственный педагогический университет им. А.И. Герцена, \\ Санкт-Петербург, Россия \\ E-mail: biomax89@yandex.ru
}

\begin{abstract}
Представлены результаты экспериментального исследования и численного моделирования динамики развития канала переключения в планарных структурах на основе диоксида ванадия. Полученные результаты по изменению со временем температуры в канале и протекающего при импульсном нагружении тока и временам перехода из высокоомного состояния в низкоомное и обратно анализируются на предмет определения механизма переключения и прогнозирования функциональных характеристик переключательных оксиднованадиевых структур как перспективных для создания релаксационных генераторов - прототипов нейроосцилляторов. Показано, что переключение связано с фазовым переходом „металл-полупроводник“ в диоксиде ванадия, стимулируемым выделением джоулева тепла.
\end{abstract}

Работа выполнена при поддержке Российского научного фонда, грант № 161900135.

DOI: $10.21883 /$ FTT.2018.03.45542.05D

\section{1. Введение}

Электрическое переключение (ЭП) в пленках диоксида ванадия, обнаруженное еще в начале 70 -х годов [1], было и остается предметом многочисленных исследований [2-12]. В работах [1-3] высказывалась идея о том, что ЭП в рассматриваемых объектах связан с присущим диоксиду ванадия фазовым переходом „металл-полупроводник“ (ФПМП). Развитию этой идеи, как и других предлагаемых моделей переключения [8-12], может способствовать установление динамики процесса.

Недостаточность знаний о процессах, определяющих процесс переключения, сдерживает достижения практически значимых результатов. В настоящее время эта проблема существенно актуализировалась в связи с интересом, проявляемым к ЭП в диоксиде ванадия как перспективному материалу для создания элементов простейших релаксационных генераторов - прототипов нейроосцилляторов (элементов осцилляторных нейронных сетей) [13,14], а также как модельному объекту исследований в нелинейной и хаотической динамике. Важно отметить, что считавшиеся недостатками свойства пленочных образцов на основе $\mathrm{VO}_{2}$ (сравнительно низкая температура ФПМП и скачок проводимости, высокий уровень токовых шумов вблизи пороговых напряжений переключения) в указанной области применения в определенном смысле обращаются в их достоинства.

Так, наличие относительно сильного шума в пороговых точках переключения является положительным фактором в связи с важной ролью эффектов коллективной синхронизации и стохастического резонанса в реальных нейронных сетях [15]. Если говорить о температурном диапазоне работы искусственной нейронной сети на основе $\mathrm{VO}_{2}$-осцилляторов, то здесь близкое к комнатной температуре значение $T_{t}$ является достоинством, поскольку она сравнима с диапазоном температур жизнедеятельности большинства биологических объектов. Для создания $R C$-осциллятора на основе $\mathrm{VO}_{2}$ переключателя, в принципе, не требуется и высокий скачок проводимости пленок при ФПМП, так как элементы можно создавать в планарных (в виде пленочных мостиков) и сандвич-конфигурациях с высокой степенью наномасштабируемости методами стандартной литографии.

Принципиально важную роль в функционировании переключателя играет его быстродействие, определяемое динамикой развития канала переключения. При том, что длительность перехода из высокоомного в низкоомное состояние определялась в ряде работ [5-11], практически отсутствуют данные о длительности обратного перехода диоксида ванадия: из низкоомного в высокоомное состояние после прохождения импульса напряжения. Единственным исключением является, по-видимому, работа $[16]$, в которой представлены результаты исследования эффекта ЭП в структурах на основе $\mathrm{NbO}_{2}$, где переключение связано с ФПМП, имеющем место в диоксиде ниобия при значительно более высокой по сравнению с $\mathrm{VO}_{2}$ температуре $(1070 \mathrm{~K})$ [17].

Для определения динамики развития канала переключения с очевидностью необходимо численное моделирование протекающих в структуре процессов, включая расчет временной зависимости температуры в канале и сопоставление его результатов с данными натурно- 

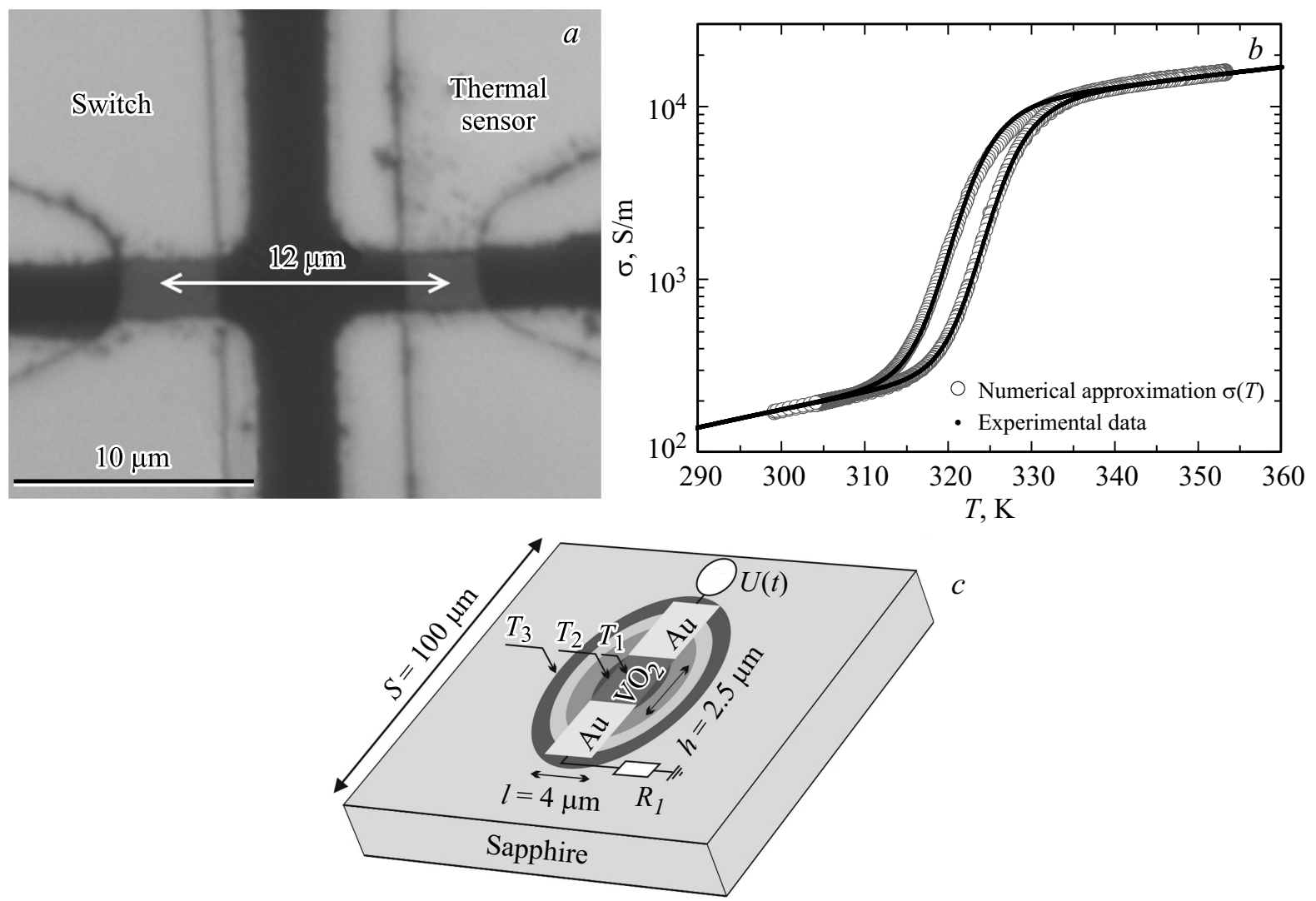

Рис. 1. Фотография планарных $\mathrm{VO}_{2}$ структур $(a)$, экспериментальная и расчетная температурные зависимости удельной проводимости пленки оксида ванадия $(b)$, область, используемая для расчета, и электрическая схема $(c)$.

го эксперимента на предмет установления механизма переключения и прогнозирования быстродействия (допустимых частот функционирования) переключательных устройств. Решение этих вопросов составляет содержание настоящей работы.

\section{2. Методика эксперимента и численного моделирования}

Пленки оксида ванадия получались методом DC магнетронного распыления по методике, описанной в [13]. В качестве диэлектрической подложки использовался $R$-cut сапфир. Толщина полученных тонких пленок оксида ванадия составила $d \sim 250 \mathrm{~nm}$. Для формирования структур проводились два этапа литографии, с использованием лазерного литографа $\mu \mathrm{PG}-101$. На первом этапе формировались участки оксидной пленки ванадия, методом травления в $4 \mathrm{~N}^{-\mathrm{HO}_{3}}$ через маску резиста. На втором этапе, методом lift-off-литографии, формировались двухслойные $\mathrm{V}-\mathrm{Au}$ контакты к переключателям, толщина слоев $\mathrm{V}$ и $\mathrm{Au}$ в которых составляли $20 \mathrm{~nm}$ и $40 \mathrm{~nm}$ соответственно. Конфигурация оксидных структур позволяла оценивать изменение температуры $\Delta T(t)$ подложки на соответствующем расстоянии от переключателя, при этом ЭП происходило только на одной структуре, другая выступала как резистивный датчик температуры. Ширина $l$ и зазор $h$ межэлектродного промежутка переключателей (рис. $1, c)$ составляли $\sim 3-4 \mu \mathrm{m}$ и $2.5 \mu \mathrm{m}$ соответственно. Полученные парные, электрически изолированные планарные микроструктуры, на расстоянии $12 \mu \mathrm{m}$, с формой контактов показаны на рис. $1, a$. После литографии производился отжиг структур на воздухе при температуре $380^{\circ} \mathrm{C}$ в течение $10 \mathrm{~min}$.

Рентгеноструктурный анализ пленки показал, что отжиг сопровождается частичным окислением и кристаллизацией пленки оксида ванадия, с образованием $\mathrm{V}_{2} \mathrm{O}_{5}, \mathrm{~V}_{2} \mathrm{O}_{3}$ и, преимущественно, $\mathrm{VO}_{2}$ фаз. Это также подтверждается температурной зависимостью проводимости пленки (рис. $1, b)$, измеренной четырехзондовым методом, на которой наблюдается скачек проводимости $\sim 10^{2}$, при температуре перехода $T_{t} \sim 320 \mathrm{~K}$. Значение $T_{t}$, как видно, меньше присущей монокристаллам диоксида ванадия $(340 \mathrm{~K})$, что характерно для пленочных образцов.

Электрическая схема, используемая для измерений показана на рис. $1, c$. Резистор $R_{i}$ служит для ограничения тока разрядки переключателя, и в зависимости от условий эксперимента и моделирования его величина варьировала в диапазоне 50-250 $\Omega$. В качестве источника напряжения и прибора для измерения малых токов использовался двухканальный источник-измеритель 
Keythley 2636А. Исследования динамики переключения проводились при помощи цифрового четырехканального осциллографа Picoscope 5442B, максимальная частота дискретизации которого составляла $125 \mathrm{MHz}$ в 14-битном режиме.

В качестве базовых при моделировании электрических и тепловых процессов в планарных микроструктурах выступали уравнения теплопроводности и непрерывности тока, которые в декартовых координатах имеют вид

$$
\left\{\begin{aligned}
\frac{\partial}{\partial t}(c T)= & \frac{\partial}{\partial x}\left(\lambda \frac{\partial T}{\partial x}\right)+\frac{\partial}{\partial y}\left(\lambda \frac{\partial T}{\partial y}\right)+\frac{\partial}{\partial z}\left(\lambda \frac{\partial T}{\partial z}\right) \\
+\sigma & {\left[\left(\frac{\partial \varphi}{\partial x}\right)^{2}+\left(\frac{\partial \varphi}{\partial y}\right)^{2}\right], } \\
\operatorname{div}(j)= & \frac{\partial}{\partial x}\left(\sigma \frac{\partial \varphi}{\partial x}\right)+\frac{\partial}{\partial y}\left(\sigma \frac{\partial \varphi}{\partial y}\right)=0,
\end{aligned}\right.
$$

где $c$ - теплоемкость, $\lambda-$ коэффициент теплопроводности, $\sigma$ - удельная электрическая проводимость, $T-$ температура, $j$ - плотность тока и $\varphi-$ потенциал. $\mathrm{B}(1)$ ввиду малой толщины пленки $\mathrm{VO}_{2}(d=250 \mathrm{~nm})$ по сравнению с длиной канала $(h=2.5 \mu \mathrm{m})$ распределение потенциала по толщине пленки считалось однородным, то есть $\partial U / \partial z=0$.

Зависимость проводимости от температуры описывалась следующей непрерывной функцией:

$$
\begin{aligned}
\sigma(T) & =\left(\frac{A_{1}-A_{2}}{1+\exp \left(\alpha_{1}\left(T-T_{c r}\right)\right)}+A_{2}\right) \\
& \times \exp \left(-\frac{1}{T}\left(\frac{B_{1}-B_{2}}{1+\exp \left(\alpha_{2}\left(T-T_{c r}\right)\right)}+B_{2}\right)\right),
\end{aligned}
$$

где $A_{1}=1.70 \cdot 10^{5} \mathrm{~S} / \mathrm{m}, A_{2}=1.53 \cdot 10^{6} \mathrm{~S} / \mathrm{m}, B_{1}=2060 \mathrm{~K}^{-1}$, $B_{2}=1622 \mathrm{~K}^{-1}, \alpha_{1}=0.5 \mathrm{~K}^{-1}, \alpha_{2}=0.3 \mathrm{~K}^{-1}, T_{c r 1}=326 \mathrm{~K}$, $T_{c r 2}=322 \mathrm{~K}$. Функция (2) хорошо соответствует прямой ветви экспериментальной зависимости проводимости пленок диоксида ванадия при $T_{c r}=T_{c r 1}$, и обратную ветвь при $T_{c r}=T_{c r 2}$ с шириной петли гистерезиса $\sim 4 \mathrm{~K}$ (см. рис. $1, b)$.

Формула (2) получена в предположении, что температурная зависимость проводимости вблизи фазового перехода может быть представлена как

$$
\sigma(T)=\Delta V(T) \sigma_{1}(T)+(1-\Delta V(T)) \sigma_{2}(T),
$$

где проводимости $\sigma_{1}(T)$ и $\sigma_{2}(T)$ проводимость полупроводниковой и металлической фаз соответственно,

$$
\Delta V(T)=\frac{1}{1+\exp \left(\frac{T-T_{c r}}{\Delta T}\right)}
$$

- сигмоидная функция Больцмана, а $\Delta T$ имела смысл эффективной ширины гистерезиса, связанной с ФПМП кристаллитов $\mathrm{VO}_{2}$, составляющих пленку $[18,19]$.
Моделирование осуществлялось для трехмерной кубической области $(100 \times 100 \times 100 \mu \mathrm{m})$ (рис. $1, c)$. На нижней и боковых границах расчетной области температура принималась равной $T_{0}=300 \mathrm{~K}$. На верхней грани, где находится пленка $\mathrm{VO}_{2}$, поперечный градиент температуры принимался равным нулю, что отвечает отсутствию теплоотвода. В начальный момент температура всей структуры принималась равной $T=T_{0}$. На электроды подавалась разность потенциалов от источника $U(t)$ через ограничительное сопротивление $R_{i}$ (см. рис. $1, c$ ). Поведение электронных свойств структуры изучалось при подаче на нее пилообразного и импульсного напряжения. Теплота, выделяющаяся при ФПМП, и эффект Пельтье на границе $\mathrm{Au}-$ электрод- $\mathrm{VO}_{2}$ не учитывались в силу их малости по сравнению с фигурирующей в переключении тепловой энергией.

Для численного моделирования использовался метод конечных разностей на неравномерной сетке с шагом, увеличивающимся с удалением от межэлектродного зазора по геометрической прогрессии $h_{i}=h \cdot(1.01)^{i}$ $(i$ - номер узла сетки) ввиду быстрого уменьшения градиентов температуры и потенциала. Для дискретизации производных с нелинейными коэффициентами использовался консервативный интегро-интерполяционный метод [20] с усреднением теплопроводности и проводимости между узлами сетки в виде $\lambda_{i+0.5}=\sqrt{\lambda_{i} \lambda_{i+1}}$ и $\sigma_{i+0.5}=\sqrt{\sigma_{i} \sigma_{i+1}}$. Численное интегрирование системы (1) по времени с шагом $\Delta t$ производилось с помощью метода, ранее использованного в [21], аналогичного методу Зейделя [22]. Значения параметров диоксида ванадия и сапфира были взяты из $[23,24]$.

\section{3. Результаты и обсуждение}

Начнем с рассмотрения поведения свойств изучаемой структуры при приложении к ней пилообразного напряжения. На рис. 2, $a$ показаны экспериментальная и расчетная вольт-амперные характеристики микроструктуры, при значении ограничительного токового резистора $R_{i}=250 \Omega$ и скорости изменения напряжения $\sim 6 \mathrm{kV} / \mathrm{s}$ (см. график $U(t)$ рис. 2,b). Видно, что результаты расчета и эксперимента практически совпадают, что свидетельствует о правомерности предлагаемой модели.

На рис. 2, $b$ приведены временные зависимости тока $I(t)$, температуры в центре $T_{1}(t)$ и на краю $T_{2}(t)$ области канала переключателя $\left(R_{i}=250 \Omega\right)$, при заданном линейно нарастающем напряжении $U(t)$. Положение контрольных точек $\left(T_{1}, T_{2}\right)$ показано на рис. $1, c$. На графиках наблюдаются резкие скачки силы тока (до $\left.I \sim 10^{-2} \mathrm{~A}\right)$ и температуры $\left(T_{1} \sim 450 \mathrm{~K}\right)$ при достижении напряжением значения $U=V_{t h}$, причем, что особенно интересно, начало роста тока соответствует моменту достижения температуры ФПМП $T_{1}=T_{t} \sim 320 \mathrm{~K}$. Далее, с увеличением $U$ ток и температура продолжают расти, максимальная температура и ее изменение относительно $T_{0}$ при напряжении $U=6 \mathrm{~V}$ coставили $T_{1 \max }=510 \mathrm{~K}\left(\Delta T_{1}=210 \mathrm{~K}\right)$ и $T_{2 \max }=480 \mathrm{~K}$ 

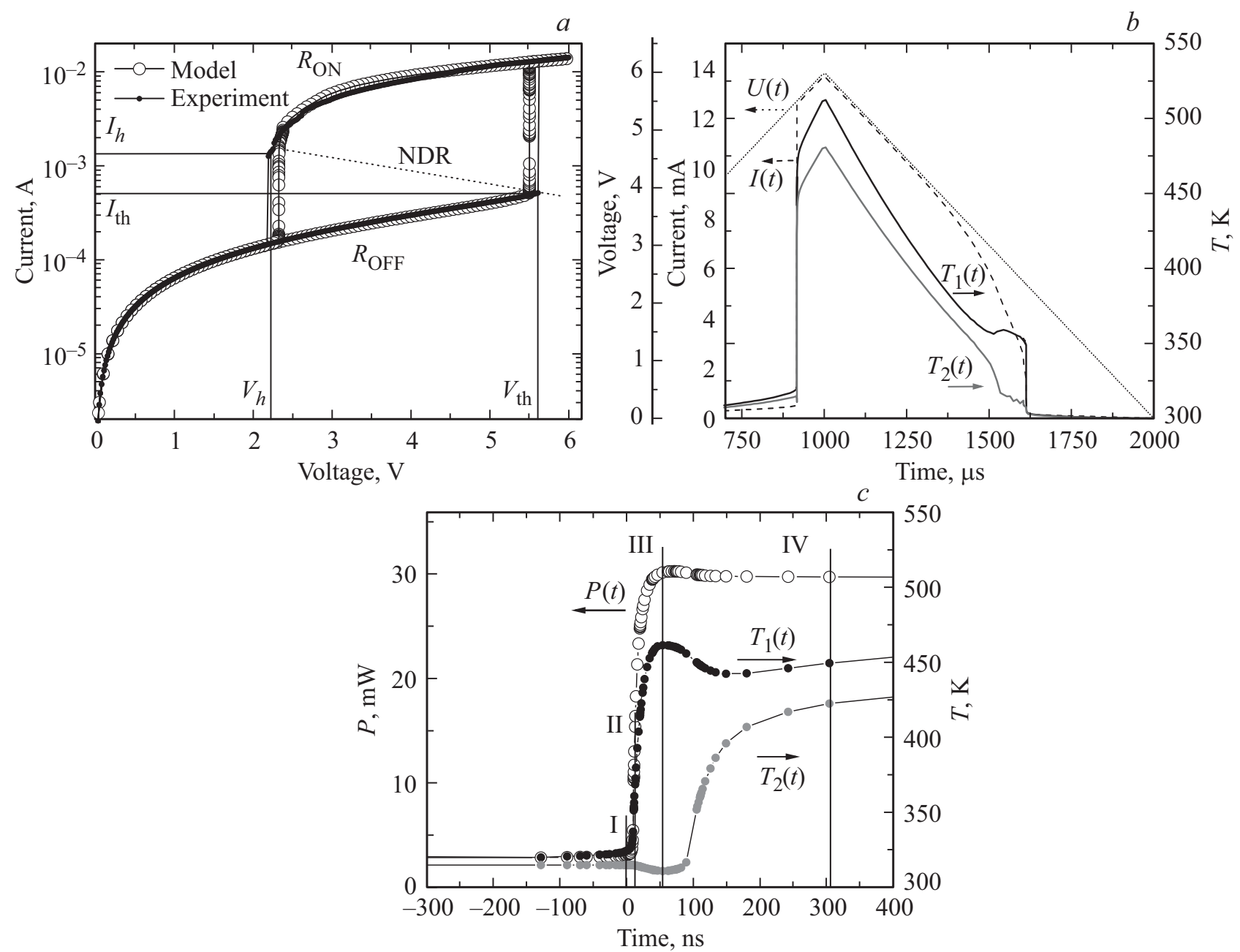

Рис. 2. а) Экспериментальная и расчетная вольт-амперные характеристики структуры при электрическом переключении, $b)$ зависимости напряжения, тока и температуры в центре канала $T_{1}$ и на краю канала $T_{2}$ от времени, $c$ ) детальный вид зависимостей температур $T_{1}, T_{2}$ и мощности $P$ от времени при переходе структуры из высокоомного в низкоомное состояние, метками (I, II, III, IV) обозначены моменты времени, отвечающие фиксации распределений температуры и плотности тока, представленных на рис. 3 .

$\left(\Delta T_{2}=180 \mathrm{~K}\right)$. Максимальная температура на периферии меньше температуры в центре канала, что связано с радиальным распределением теплового потока в сапфировой подложке от, условно, точечного источника. Последнее утверждение верно в предположении, что токовый шнур образуется в центре канала.

Моделирование переключения с использованием меньшего по значению ограничительного резистора $\left(R_{i}=50 \Omega\right)$ показало, что температура $T_{1 \max }$ может достигать $850 \mathrm{~K}$. Этот факт означает, что за микросекундные времена разрядки конденсатора в осцилляторах [4] пленка $\mathrm{VO}_{2}$ при таких температурах может модифицироваться - на воздухе происходить окисление (рост $R_{\mathrm{OFF}}$ ), а в вакууме восстановление (снижение $\left.R_{\mathrm{OFF}}\right)$, подобно эффектам электронно-лучевой модификации, наблюдаемым в работе [25]. По нашему мнению, модификация, индуцированная повышенной температурой канала переключения, является одним из основных факторов нестабильности переключательных структур и невысокого срока службы. Также стоит отметить, что в ходе переключения при резких нагревах межэлектродного промежутка из-за наличия разницы в коэффициентах линейного расширения материалов и локальности нагрева, в данной области могут возникать термоиндуцированные механические напряжения. При недостаточной адгезии эти напряжения могут вызывать отслаивания как металлических контактов от пленки $\mathrm{VO}_{2}$, так и самой пленки от подложки.

При более детальном анализе возрастания температуры, при образовании токового канала, видны особенности ее поведения (рис. 2,c). Видно, что возрастание температуры (и тока) происходит очень резко, за времена $\tau_{f} \sim 20-30 \mathrm{~ns}$, при этом фронт температуры имеет максимум, образующийся на временах $\sim 60 \mathrm{~ns}$ с высотой пика $\delta T_{1} \sim 20 \mathrm{~K}$. Образование последнего обусловлено, по-видимому, пиковым возрастанием мощности $P(t)$, выделяемой в канале, связанным с перераспределением напряжения между токовым резистором и переклю- 

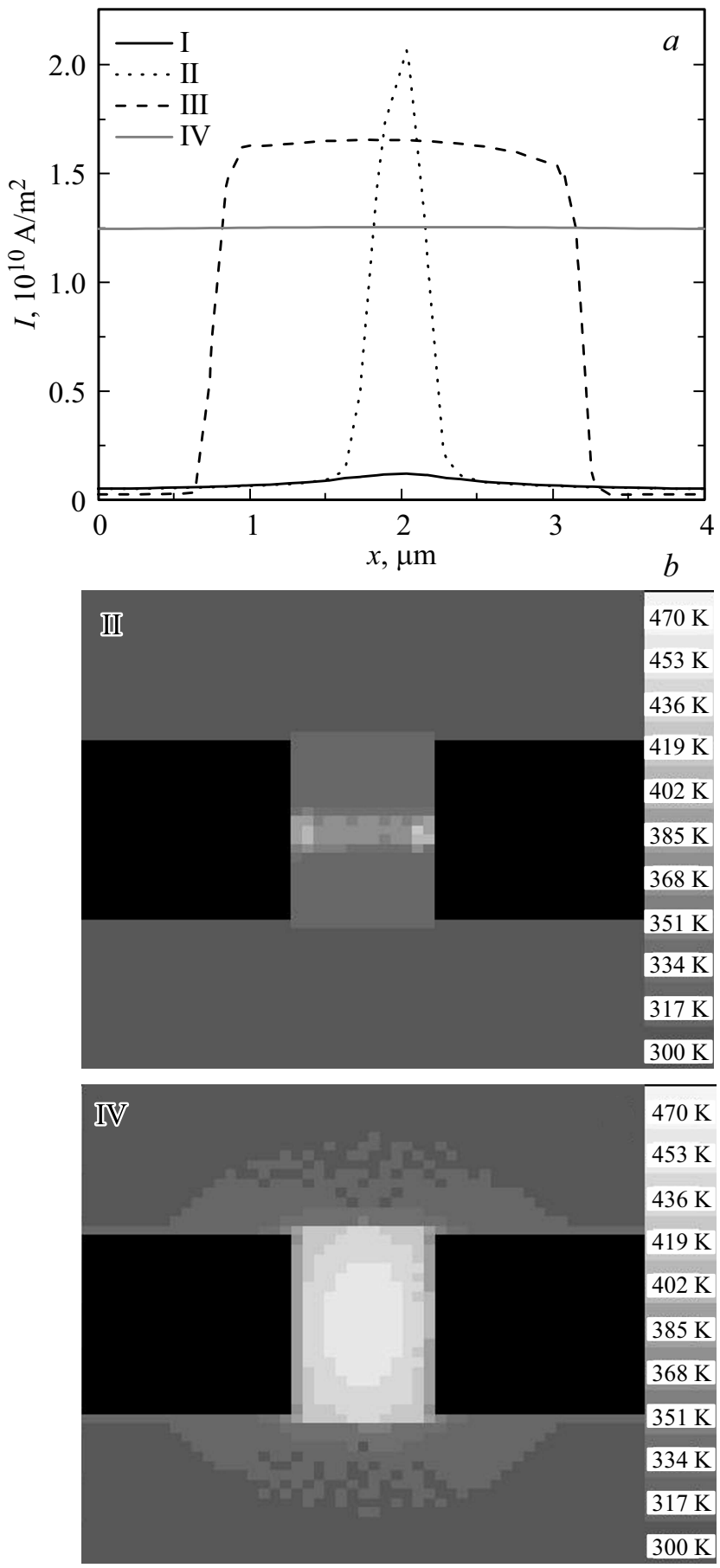

Рис. 3. a) Распределение плотностей тока в различные моменты времени, указанные на рис. 2, $c, b)$ расчетные распределения температуры в моменты (II) и (IV).

чателем, что заметно увеличивает плотность тока в этот момент. Минимум на зависимости $T_{2}(t)$ связан с перераспределением плотности тока по сечению.

На рис. 3 в виде расчетных распределений температуры и плотности тока показано формирование канала переключения в изучаемой структуре. Видно, что в начальный период (I) перед образованием токового шнура, распределение плотности тока имеет слабый максимум, а температура достигает температуры ФПМП. Далее происходит шнурование тока (II), с образованием проводящего канала шириной $\sim 500 \mathrm{~nm}$. Затем токовый канал начинает расширяться (III), при этом температура в центре достигает максимального значения (рис. 2,c) и минимальна на периферии, где существенно понижена плотность тока. Далее происходит монотонный рост температуры в канале, что приводит к переходу вещества в межэлектродном пространстве в металлическое состояние.

Во время линейного снижения напряжения в определенный момент $(1400-1600 \mu \mathrm{s}$, рис. $2, b)$ ток начинает быстро снижаться до значения $I_{h}$, когда наблюдается обратное переключение $\mathrm{VO}_{2}$ в полупроводниковое состояние. Падение температуры в центре $T_{1}(t)$ на данном отрезке наоборот замедляется (рис. 2,b) и в диапазоне $1500-1600 \mu \mathrm{s}$ демонстрирует локальный максимум. Этот эффект может быть связан с происходящим вновь образованием канала металлической фазы $\mathrm{VO}_{2}$. Пониженное значение температуры на периферии $\mathrm{VO}_{2}$ пленки $T_{2}$ приводит к более раннему переходу этой области в полупроводниковое состояние (при $\sim 1500 \mu \mathrm{s}$ ), после чего температура $T_{2}$ поддерживается на некотором уровне за счет выделяемого тепла в центре канала, до момента полного выключения переключателя.

Далее рассмотрим динамику развития канала переключения в планарных $\mathrm{VO}_{2}$ структурах в импульсном режиме. Здесь мы применили оригинальный подход, основанный на использовании комбинированного внешнего сигнала. Последний представлял собой суперпозицию постоянного смещения $V_{0}$ и прямоугольного импульса длительностью варьируемой в интервале $5 \mathrm{~ns}-1 \mathrm{~ms}$. Суммарное напряжение $V_{p}$ составляло $5.6-7 \mathrm{~V}$ (см. рис. 4,5). Применение комбинированного сигнала позволяет регистрировать изменение тока и соответственно проводимости после прохождения импульса.

На рис. 4 показаны экспериментальные осциллограммы тока и напряжения при подаче комбинированного импульса на структуру. Как видно, в импульсном режиме выявляются временные параметры процесса развития канала переключения: время включения $\tau_{\text {оn }}$ и время выключения $\tau_{o f f}$. Последнее определяется как протяженность токового „хвоста“, наблюдаемого после прохождения импульса. Наблюдаемое $\tau_{\text {оn }}$ может быть представлено как сумма двух составляющих $\left(\tau_{o n}=\tau_{d e l}+\tau_{f}\right)$, где $\tau_{d e l}$ и $\tau_{f}$ времена, необходимые для достижения переключения и нарастания фронта токового импульса при переключении соответственно.

На рис. 5, а показаны экспериментальные осциллограммы тока в моменты включения и выключения переключателя, в зависимости от амплитуды импульса напряжения $V_{p}$. На рис. 5, $b$ приведены зависимости $\tau_{o n}$ и $\tau_{o f f}$ от $V_{p}$. Обращает на себя внимание наличие линейной зависимости $\tau_{o f f}\left(V_{p}\right)$. Другие факты, нуждающиеся в объяснении, состоят в росте $\tau_{o f f}$ с увеличением $V_{0}$ (рис. 6,a) и выходе $\tau_{\text {off }}$ на насыщение с возрастанием длительности импульса (рис. $6, b$ ). 


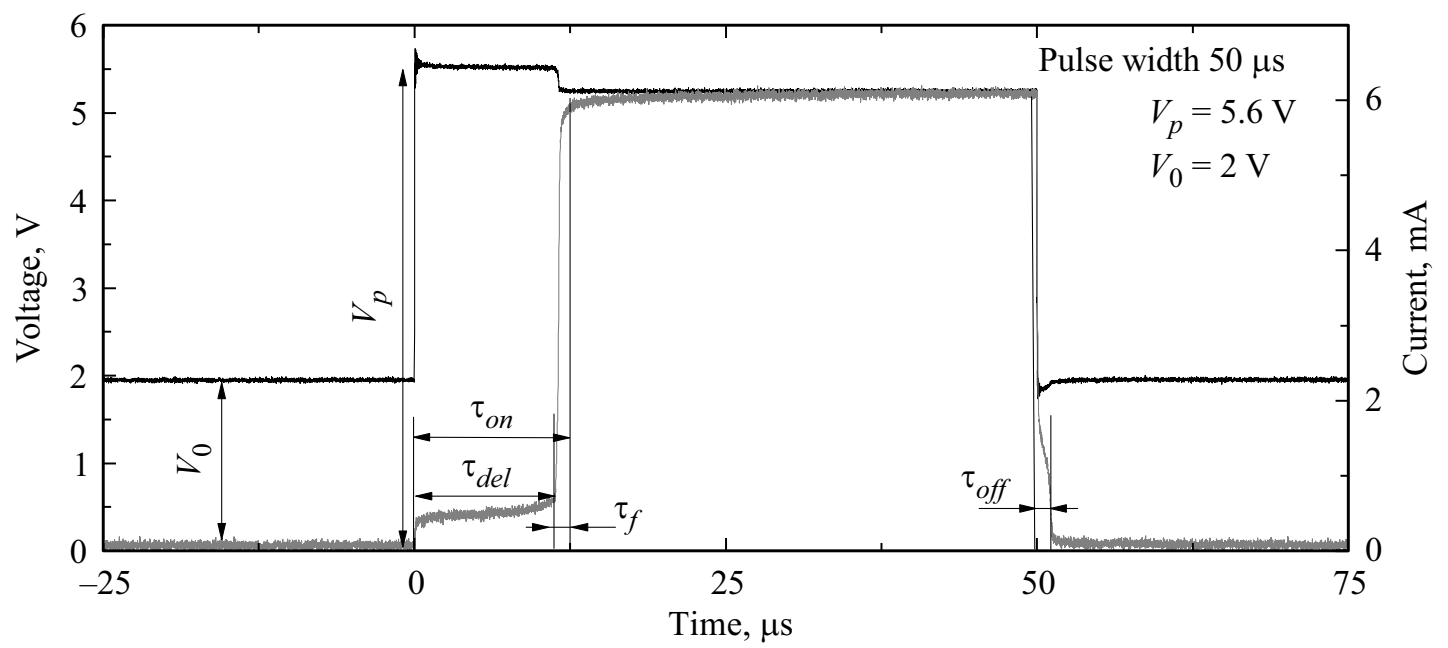

Рис. 4. Экспериментальные осциллограммы тока и напряжения при подаче комбинированного сигнала на планарную переключательную структуру.
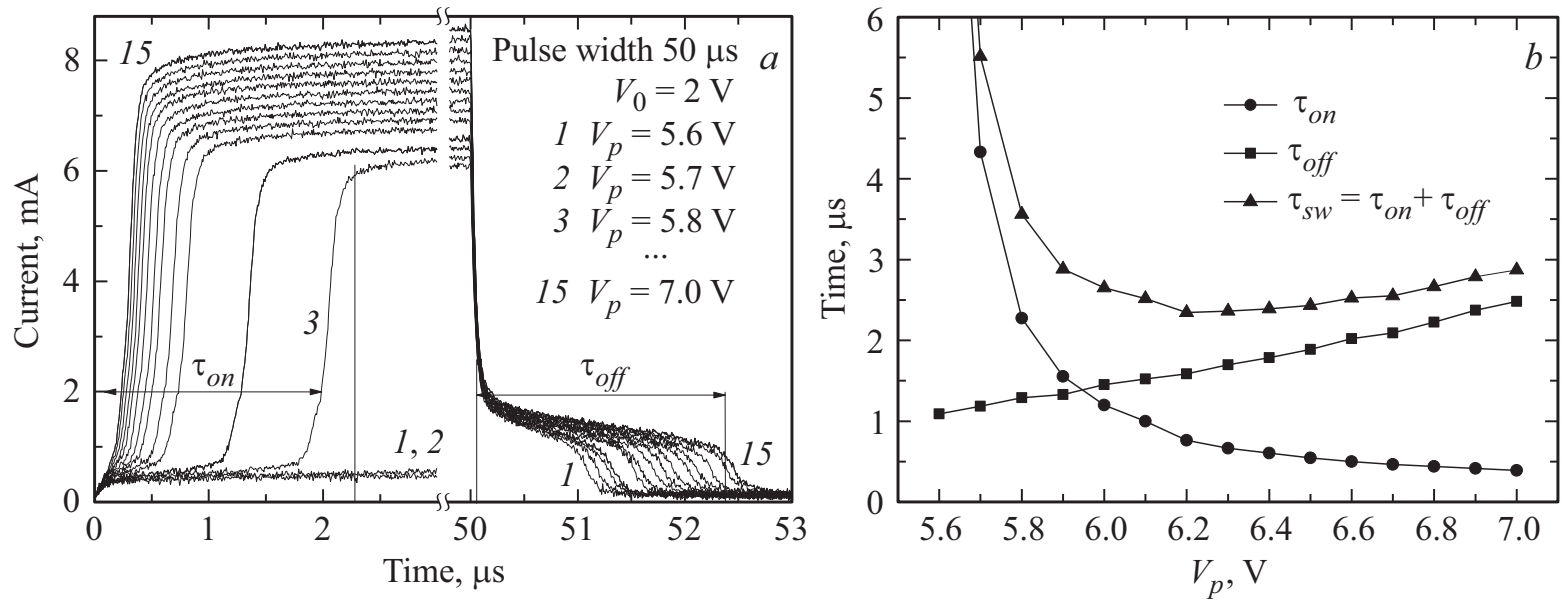

Рис. 5. Временные осциллограммы тока $(a)$ и зависимости времен включения $\tau_{\text {on }}$ и выключения $\tau_{\text {off }}$ от суммарного напряжения (опорное напряжение $\left.V_{0}=2 \mathrm{~V}\right)(b)$.
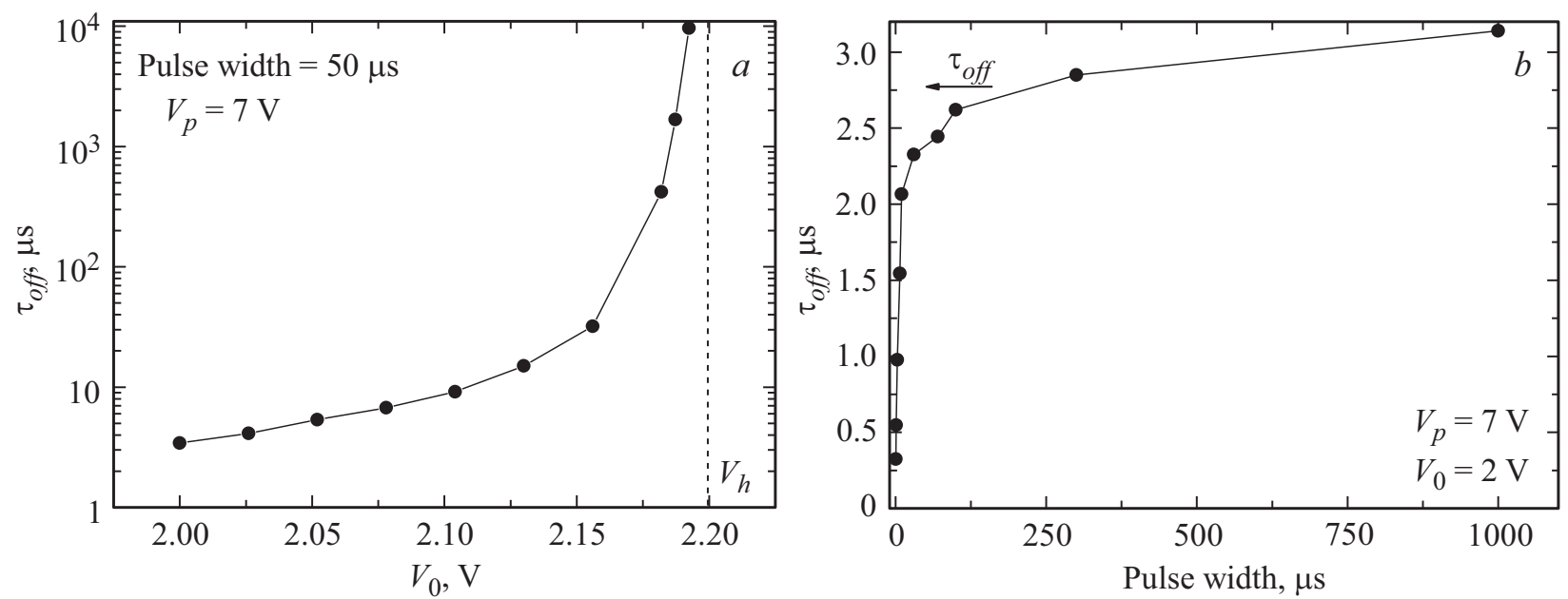

Рис. 6. a) Экспериментальная зависимость времени выключения $\tau_{\text {off }}$ от величины постоянного смещения $V_{0}$, пунктирной линией отмечено значение напряжения, при котором происходит переход структуры из низкоомного в высокоомное состояние $V_{h}$, $b)$ зависимость времени выключения $\tau_{o f f}$ от длительности импульса. 

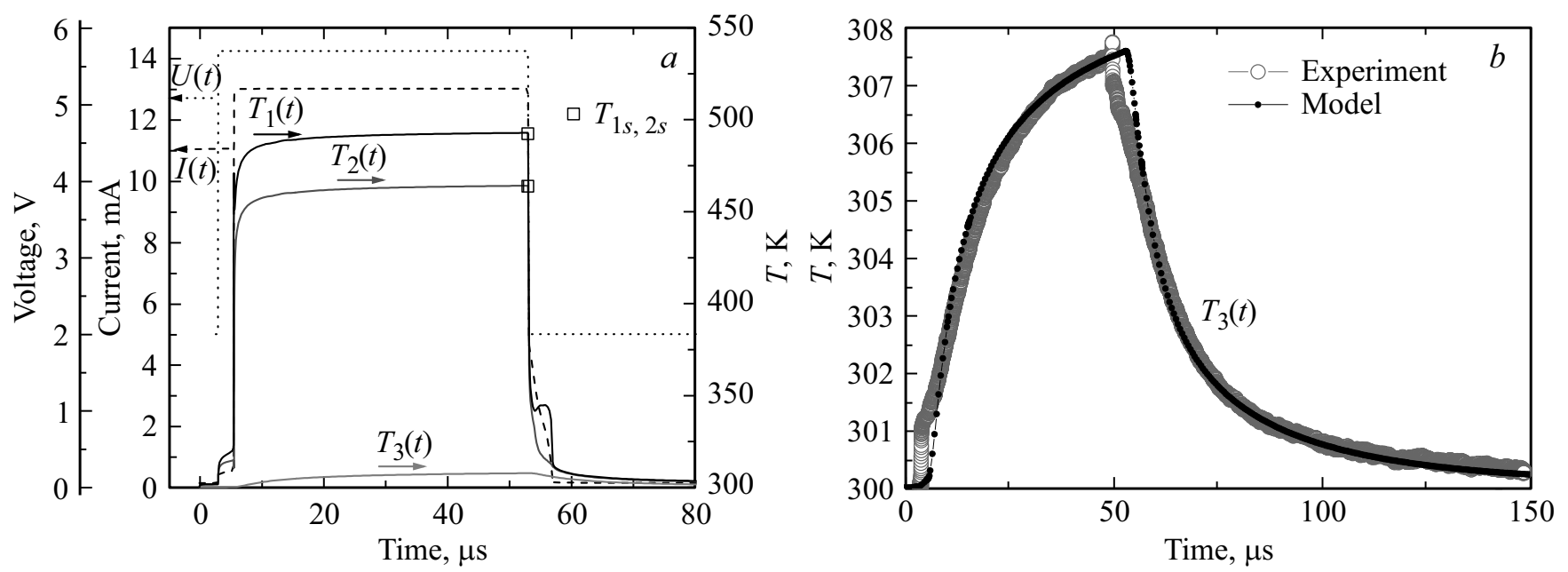

Рис. 7. a) Расчетные зависимости температуры в центре канала $T_{1}$, на краю канала $T_{2}$ и на расстоянии $12 \mu \mathrm{m}$ от него $T_{3}$ от времени (меткой указаны температуры насыщения перед выключением), $b$ ) экспериментальная и расчетные временные зависимости $T_{3}$ в импульсном режиме.

Время включения $\tau_{\text {оn }}$ обнаруживает спад с ростом $V_{p}$, причем $\tau_{f}$ изменяется не значительно, тогда как зависимость $\tau_{d e l}\left(V_{p}\right)$ имеет экспоненциальный характер и асимптотой является $V_{p}=V_{t h}$.

Сумма времен включения и выключения определяет минимальную длительность переключения $\tau_{s w}$ из неметаллического состояния в металлическое и обратно $\tau_{s w}=\tau_{o n}+\tau_{o f f}$. Эта величина имеет физический смысл минимального эффективного времени полного цикла переключения (OFF-ON-OFF), и определяет предельное время и частоту функционирования переключателя. Следует отметить интересный факт, что $\tau_{s w}$ имеет минимум при определенном значении амплитуды $V_{p}$ (рис. 5, $b$ ), разделяя условно области влияния процесса включения (нагрева) и выключения (остывания) $\mathrm{VO}_{2}$ канала на величину $\tau_{s w}$.

На рис. 7, а приведены расчетные временные зависимости напряжения, тока и температур $\left(T_{1}(t), T_{2}(t)\right)$ в импульсном режиме. Поведение выявляемых здесь $\tau_{\text {on }}$ и $\tau_{o f f}$ в зависимости от параметров комбинированного сигнала соответствует определенному экспериментально, что является еще одним указанием в пользу адекватности результатов численного моделирования.

Анализируя форму температурных зависимостей $T_{1}(t), T_{2}(t)$ можно заметить сильный скачок температуры в момент включения структуры. При этом, в отличие от пилообразного импульса, рассмотренного выше, температура стремится к насыщению $T_{1 s}=493 \mathrm{~K}$ и $T_{2 s}=464 \mathrm{~K}$. На переднем и заднем фронтах зависимости $T_{1}(t)$, как и в случае пилообразного сигнала $U(t)$, наблюдаются локальные максимумы температуры (длительностью $\sim 60 \mathrm{~ns}$ и высотой пика $\delta T_{1} \sim 20 \mathrm{~K}$ ), связанные с повышенной плотностью тока и выделяемой мощности в момент образования и схлопывания канала металлической фазы $\mathrm{VO}_{2}$. Важно отметить, что вид распределения температуры и форма канала при импульсном нагружении аналогичны представленным на рис. 3 .

На рис. 6, $b$ сопоставлены расчетная и экспериментальная временные зависимости температуры $T_{3}(t)$ (рис. $1, c)$. Как уже указывалось в разделе „Методика эксперимента“, для экспериментального измерения $T_{3}(t)$ на соответствующей дистанции от центра $(12 \mu \mathrm{m})$ размещалась такая же структура (рис. $1, a$ ), которая служила температурным датчиком. Как видно из рисунка, расчетная и экспериментальная временные зависимости $T_{3}(t)$ хорошо совпадают, что является еще одним подтверждением адекватности численной модели. Формы всех трех временных зависимостей $T_{1}(t), T_{2}(t)$ и $T_{3}(t)$ существенно отличаются, что и понятно, т.к. они соответствуют разным расстояниям до центра канала, при этом на $T_{2}(t)$ и $T_{3}(t)$ нет локальных максимумов, соответствующих образованию и схлопыванию канала, а $T_{3}(t)$ не выходит на насыщение за время действия импульса, так как для нагрева широкой области подложки нужны импульсы гораздо большей длительности. Максимальное значение $T_{3}$ не превышает $308 \mathrm{~K}$, то есть перегрев относительно подложки не выше $8 \mathrm{~K}$, что может влиять на соседние микроструктуры и являться причиной возникновения эффекта термосвязи между переключателями и осцилляторами на их основе, который описан нами в работе [26].

Возвращаясь к факту наличия насыщения $\tau_{\text {off }}$ с ростом длительности импульса, отметим, что он может быть объяснен насыщением $T_{1}$ в течение действия импульса (очевидно, что $\tau_{o f f}$ пропорционально $T_{1 s}$ ). При коротких импульсах зависимость $\tau_{o f f}$ от длительности импульса также соответствует зависимости $T_{1}(t)$.

Причина увеличения $\tau_{o f f} \mathrm{c}$ ростом $V_{0}$ становится понятной если проанализировать расчетные временные зависимости тока и температуры при опорном напряжении, отвечающем большим значениям $\tau_{\text {off }}$ (например, 


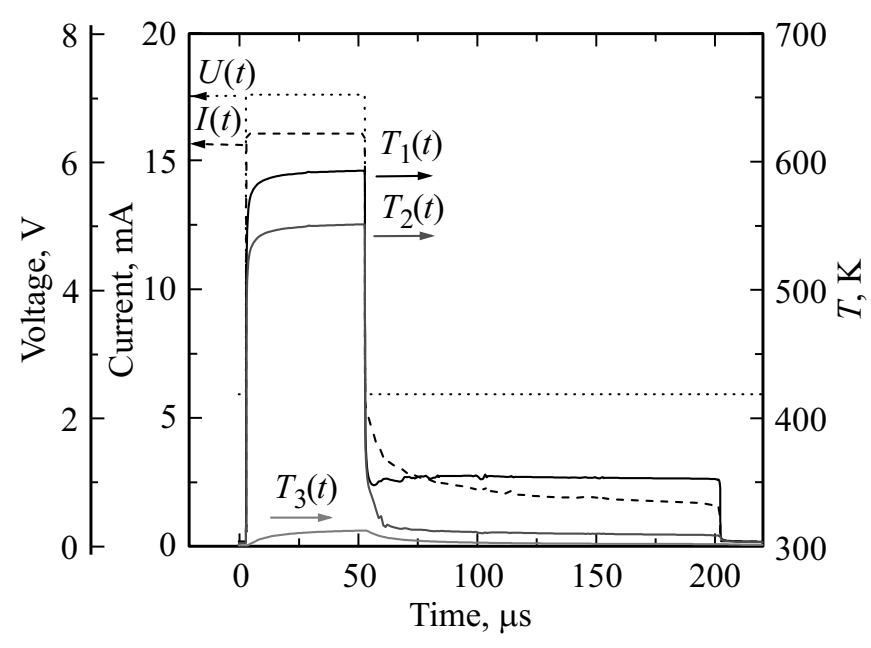

Рис. 8. Расчетные зависимости температуры в центре канала $T_{1}$, на краю канала $T_{2}$ и на расстоянии $12 \mu \mathrm{m}$ от него $T_{3}$ от времени при $V_{0}=2.3 \mathrm{~V}$.

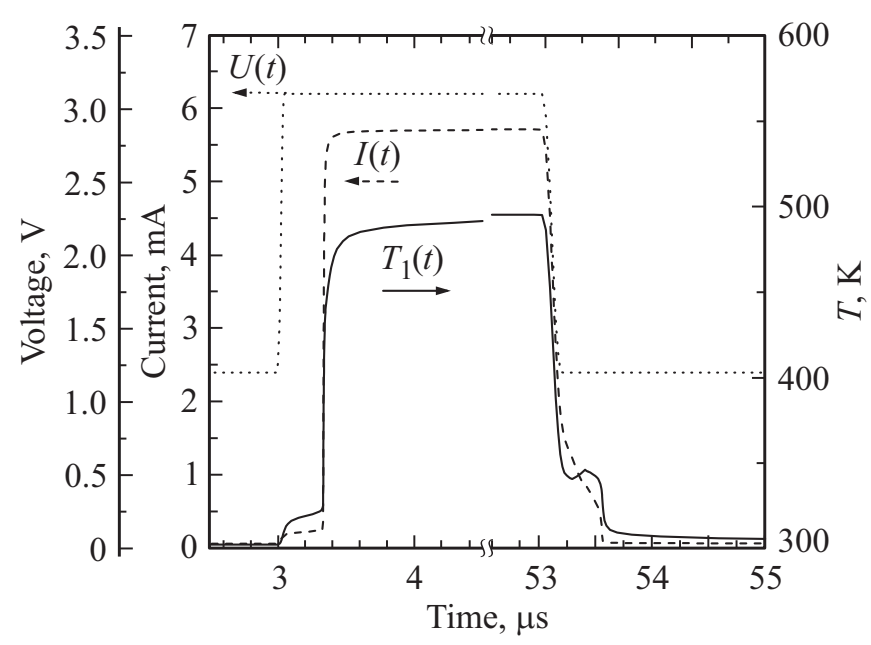

Рис. 9. Расчетные зависимости тока и температуры в центре канала $T_{1}$ для переключательной структуры с активной областью размерами $1.2 \times 0.8 \mu \mathrm{m}$.

как это сделано в работе при $V_{0}=2.3 \mathrm{~V}$ и соответственно $\tau_{o f f} \sim 150 \mu \mathrm{s}-$ рис. 8). По зависимости $T_{1}(t)$ видно, что в данном случае образуется квазистабильный токовый канал, с примерно постоянной по времени температурой $T_{1} \sim 350 \mathrm{~K}$, который поддерживается током за счет повышенного значения $V_{0}$. Таким образом, $V_{0}$ влияет на время остывания структуры и при приближении $V_{0} \rightarrow V_{h}$ (расчетное $V_{h}=2.35 \mathrm{~V}$, экспериментальное $V_{h}=2.2 \mathrm{~V}$ ), токовый канал (металлической фазы) становится устойчивым и может поддерживаться неограниченно долгое время. По распределению температуры области остывания можно увидеть, что канал имеет вид шнура с формой подробно изображенной на рис. $3, a$. В отношении зависимостей $T_{2}(t)$ и $T_{3}(t)$ отметим, что они практически не меняются при вариации $V_{0}$, т.к. ширина токового канала значительно меньше ширины электродов и выделяемая мощность незначительна. Также не меняется значение температуры насыщения $T_{1 s}$, которая зависит от $V_{p}$ и длительности импульса.

Таким образом, время остывания канала можно условно разбить на две составляющие, базисную и дополнительную $\tau_{o f f}=\tau_{o f f 1}+\tau_{o f f 2}$. Базисная составляющая $\tau_{o f f 1}$ обусловлена остыванием канала до температур близких к температуре перехода $\mathrm{VO}_{2}$ из неметаллического в металлическое состояние $\sim 350 \mathrm{~K}$. Эта составляющая присутствует всегда и определяется температурой $T_{1 s}$. При данной конфигурации переключателя $\tau_{o f f 1}$ не превышает единиц микросекунд $(1-3 \mu \mathrm{s})$, что определяет минимальный предел $\tau_{o f f}$. В свою очередь дополнительная составляющая $\tau_{o f f 2}$ присутствует только при наличии высокой постоянной составляющей $V_{0}$, и может меняться в очень широких пределах (рис. 6).

При величинах напряжения смещения в диапазоне от $V_{h}$ до $V_{t h}$ структуру можно перевести во включенное состояние, сохраняющееся длительное время, и обратно импульсом обратной полярности, реализовав тем самым эффект резистивного переключения с памятью.

В заключении проанализируем, какие изменения происходят при уменьшении геометрических размеров переключателя. На рис. 9 показаны расчетные зависимости $I(t)$ и $T_{1}(t)$, для структуры с размерами $1.2 \times 0.8 \mu \mathrm{m}$, и соответственно площадью на порядок меньшей по отношению к ранее рассмотренной. Толщина пленки и $T_{1 s}$ оставались прежними. Как показали полученные результаты, с уменьшением площади переключателя время выключения $\tau_{o f f}$ уменьшается практически на порядок и составляет $\tau_{o f f} \sim 600$ ns. Аналогично уменьшается и время включения $\tau_{\text {оn. }}$. Таким образом, с уменьшением размеров переключателя развитие электрического переключения ускоряется.

\section{4. Заключение}

В результате проведенных экспериментальных исследований и численного моделирования динамики развития канала переключения в планарных структурах на основе диоксида ванадия установлены закономерности поведения времен перехода из высокоомного в низкоомное состояние и обратно в зависимости от амплитуды и длительности импульса и величины опорного напряжения. Полученные данные в совокупности с результатами расчета и измерений температуры в канале переключения свидетельствуют об определяющей роли в процессе переключения фазового перехода „металл-полупроводник“ в диоксиде ванадия, стимулированного джоулевым разогревом. Показано, что характерная для пленочных структур деградация в процессе многократных переключений может быть существенно снижена посредством ограничения тока. 
Полученные результаты дают основание для прогнозирования допустимых частот функционирования переключательных устройств и их оптимизации в плане быстродействия. В частности, показано, что быстродействие может быть существенно повышено при уменьшении геометрических размеров активной области переключателя. Для дальнейших исследований представляет интерес влияние на процесс переключения эффектов сильного поля.

\section{Список литературы}

[1] А.А. Бугаев, Б.П. Захарченя, Ф.А. Чудновский. Фазовый переход полупроводник-металл и его применение. Наука, Л. (1979). 183 c.

[2] Б.П. Захарченя, В.П. Малиненко, Г.Б. Стефанович, М.Ю. Терман, Ф.А. Чудновский. Письма в ЖТФ 11, 2, 108 (1985).

[3] F.A. Chudnovskii, L.L. Odynets, A.L. Pergament, G.B. Stefanovich. J. Solid State Chem. 122, 95 (1996).

[4] A. Pergament, G. Stefanovich, V. Malinenko, A. Velichko. Adv. Condens. Matter Phys. 2015, 26 (2015).

[5] D. Li, A.A. Sharma, D.K. Gala, N. Shukla, H. Paik, S. Datta, D.G. Schlom, J.A. Bain, M. Skowronski. ACS Appl. Mater. Interfaces 8, 20, 12908 (2016). DOI: 10.1021/acsami.6b03501

[6] G.M. Liao, S. Chen, L.L. Fan, Y.L. Chen, X.Q. Wang, H. Ren, Z.M. Zhang, C.W. Zou. AIP Advances 6, 045014 (2016).

[7] I.P. Radu, B. Govoreanu, S. Mertens, X. Shi, M. Cantoro, M. Schaekers, M. Jurczak, S. De Gendt, A. Stesmans, J.A. Kittl, M. Heyns, K. Martens. Nanotechnology 26, 165202 (2015).

[8] J.K. Higgins, B.K. Temple, J.E. Lewis. J. Non-Cryst. Solids 23, 87 (1977).

[9] A.B. Pevtsov, A.V. Medvedev, D.A. Kurdyukov, N.D. Il'inskaya, V.G. Golubev, V.G. Karpov. Phys. Rev. B 85, 024110 (2012).

[10] G. Gopalakrishnan, D. Ruzmetov, S. Ramanathan. J. Mater. Sci. 44, 5345 (2009).

[11] B.-G. Chae, H.-T. Kim, D.-H. Youn, K.-Y. Kang. Physica B Condens. Matter 369, 76 (2005).

[12] E. Janod, J. Tranchant, B. Corraze, M. Querré, P. Stoliar, M. Rozenberg, T. Cren, Dimitri Roditchev, V. Ta Phuoc, M.-P. Besland, L. Cario. Adv. Funct. Mater. 25, 6287 (2015).

[13] A. Velichko, M. Belyaev, V. Putrolaynen, A. Pergament, V. Perminov. Int. J. Modern Phys. 31, 1650261 (2017).

[14] D. Vodenicarevic, N. Locatelli, F. Abreu Araujo, J. Grollier, D. Querlioz. Sci. Rep. (2017). DOI: 10.1038/srep44772.

[15] Г.Н. Борисюк, Р.М. Борисюк, Я.Б. Казанович, Г.Р. Иваницкий. УФН 172, 1189 (2002).

[16] G.C. Vezzoli. J. Appl. Phys. 50, 6390 (1979).

[17] Y. Zhou, S. Ramanathan. Critical Rev. Solid State Mater. Sci. 38, 286 (2013).

[18] P. Uhd Jepsen, B.M. Fischer, A. Thoman, H. Helm, J.Y. Suh, René Lopez, R.F. Haglund,jr. Phys. Rev. B 74, 205103 (2006).

[19] И.А. Хахаев, Ф.А. Чудновский, Е.Б. Шадрин. ФТТ 36, 1643 (1994).
[20] Н.М. Беляев, А.А. Рядно. Методы теории теплопроводности. Высш. шк., М. (1982). Ч. І. 327 с.

[21] В.И. Сысун, И.В. Сысун, П.П. Борисков. ЖТФ 45, 23 (2016).

[22] В.К. Саульев. Интегрирование уравнений параболического типа методом сеток. Изд-во физ.-мат. лит. М. (1960). 324 с.

[23] E.R. Dobrovinskaya, L.A. Lytvynov, V. Pishchik. Sapphire: Material, Manufacturing, Applications. Springer Science \& Business Media (2009). 480 p.

[24] E. Freeman, A. Kar, N. Shukla, R. Misra, R. Engel-Herbert, D. Schlom, V. Gopalan, K. Rabe, S. Datta. 70th Device Research Conference. University Park, USA (2012). 243 p.

[25] M.A. Belyaev, V.V. Putrolaynen, A.A. Velichko, G.B. Stefanovich, A.L. Pergament. Jpn. J. Appl. Phys. 53, 111102 (2014).

[26] A.A. Velichko, M.A. Belyaev, V.V. Putrolaynen, V.V. Perminov, A.L. Pergament. Solid State Electronics (2017). In Press. 